\title{
Constrained balancing of two industrial rotor systems: Least squares and min-max approaches
}

\author{
Bin Huang ${ }^{\mathrm{a}}$, Daiki Fujimura ${ }^{\mathrm{b}}$, Paul Allairec ${ }^{\mathrm{c}}$, Zongli Lin ${ }^{\mathrm{a}, *}$ and Guoxin $\mathrm{Li}^{\mathrm{c}}$ \\ ${ }^{a}$ Charles L. Brown Department of Electrical and Computer Engineering, University of Virginia, Charlottesville, VA \\ 22904-4743, USA \\ ${ }^{\mathrm{b}}$ Commissioning \& Testing Section, Power Plant Construction Department, Takasago Machinery Works, \\ Mitsubishi Heavy Industries, Ltd., 2-1-1 Shinhama Arai-cho Takasago Hyogo, 676-8686, Japan \\ ${ }^{\mathrm{c}}$ Department of Mechanical and Aerospace Engineering, University of Virginia, Charlottesville, VA 22904-4746, \\ USA
}

Received 5 April 2007

\begin{abstract}
Rotor vibrations caused by rotor mass unbalance distributions are a major source of maintenance problems in highspeed rotating machinery. Minimizing this vibration by balancing under practical constraints is quite important to industry. This paper considers balancing of two large industrial rotor systems by constrained least squares and min-max balancing methods. In current industrial practice, the weighted least squares method has been utilized to minimize rotor vibrations for many years. One of its disadvantages is that it cannot guarantee that the maximum value of vibration is below a specified value. To achieve better balancing performance, the min-max balancing method utilizing the Second Order Cone Programming (SOCP) with the maximum correction weight constraint, the maximum residual response constraint as well as the weight splitting constraint has been utilized for effective balancing. The min-max balancing method can guarantee a maximum residual vibration value below an optimum value and is shown by simulation to significantly outperform the weighted least squares method.
\end{abstract}

\section{Introduction}

For high speed rotating machinery, manufacturing procedures are subject to errors in geometric dimensions and irregularities in the mass distribution due to imperfect manufacturing techniques. The resulting mass imbalance forces are a major source of rotor vibrations. As the operating speed of modern rotating machinery becomes higher and higher, balancing becomes an essential step to ensuring operational reliability. Mechanical balancing involves the addition or removal of controlled amounts of weight at certain angular and axial locations on a rotor to minimize the vibrations. These balancing methods with trial weights can be classified into two main groups [1]: modal balancing methods and influence coefficient (IC) balancing methods.

The principle of the modal balancing methods is to balance rotors using the system modal shapes, assuming them to be planar. However, the selection of the trial masses for correcting specific modes requires accurate analytical models of rotor-bearing systems [1] and, as a result, these methods are not widely used. The influence coefficient methods require only the vibration responses at different trial weights, making it relatively easy to apply in field balancing [1]. Many researchers have contributed in the development of the influence coefficients methods as shown in the 1998 review paper on rotor balancing [2].

\footnotetext{
*Corresponding author. Tel.: +1 434924 6342; Fax: +1 434924 8818; E-mail: zl5y@ virginia.edu.
} 
The first IC balancing framework was developed by Goodman [3], who noticed that the original least squares (LS) solution is not optimal in terms of the maximal residual vibration. An iterative procedure using the weighted least squares method was proposed to reduce the maximum residual vibration. However, there is no guarantee that Goodman's iterative process will converge to an optimal solution, which is the min-max solution. Little and Pilkey [4] adopted a linear programming (LP) approach and formulated the min-max IC balancing problem as a constrained optimization. The constraint that the number of balance planes exceeds the number of observations greatly limits its applications. As the optimization techniques evolve, Woomer and Pilkey later proposed a more general min-max balancing method by applying quadratic programming approach [5]. However, in both [4,5], the nonlinear balancing problem is solved approximately.

Kanki et al. [6] employed a linear matrix inequality (LMI) method from control theory to solve the balancing problem. LMI optimization is based on semidefinite programming (SDP), which is very general but not as efficient as one of its special cases, the second order cone programming [7]. Li et al. [8] used the second order cone programming (SOCP) formulation to solve the least square and min-max balancing problems. Recently, several analytical methods (see, for example [9]), without trial weights, have been proposed in the literature. The analytical methods have the advantage of requiring no trial runs or fewer trial runs. However, these methods require very well calibrated rotor models that often are not available in industrial applications. At this time, the influence coefficient methods dominate field balancing of high speed industrial rotors.

This paper reports on the calculated balancing results of two rotor systems from Mitsubishi Heavy Industrials Ltd: a gas turbine and a nuclear steam turbine. We will report the balancing results by both the least squares and min-max methods. These results were calculated based on the influence coefficients. Special attention will be given to the treatment of practical balancing constraints that are not considered in [8]. These results will indicate that the min-max balancing significantly outperforms the least square method and leads to better balancing results than those of the least squares balancing that was actually implemented at commercial power plants.

This paper is organized as follows. Section 2 briefly describes the two industrial balancing cases to be considered in this paper. Section 3 introduces the balancing methods used in this paper. Section 4 describes the balancing constraints. Section 5 summarizes the balancing results of these two different balancing cases and compares them with the actual balancing results from commercial power plants. Section 6 draws a brief conclusion to the paper. Finally, the influence coefficient framework for balancing and the formulation of the second order cone programming are summarized in the appendix.

\section{Industrial balancing applications}

In this section, we first introduce the two industrial balancing cases originating from Mitsubishi Heavy Industrials Ltd, one gas turbine and the other, a nuclear turbine. Balancing is an essential step to ensuring the safe operation and good maintenance on both pieces of large size equipment of power generation, in which some practical balancing constraints need to be strictly satisfied. The detailed information and practical constraints of these two balancing cases are described in the following two subsections separately.

\subsection{Gas turbine}

The configuration of the gas turbine we consider is shown in Fig. 1. This gas turbine has a mass of $727,000 \mathrm{Kg}$ and is $61.65 \mathrm{~m}$ long.

This gas turbine has three planes (BZ-A, BZ-C and BZ-E) available for balancing. However, in most cases, only two planes (BZ-A and BZ-E) are used because of the difficulty of placing weights in plane BZ-C. The objective of balancing here is to minimize the maximum vibration amplitude of the rotor at the measuring planes by using balance weights placed in planes BZ-A and BZ-E.

For this case, the only available balance weight type for balance planes BZ-A and BZ-E is 142gr. Each balance plane also has a limited number of weight holes, as shown in Fig. 1 (Plane BZ-A has balance holes placed every 7.5 degrees, and plane BZ-E has balance holes placed every 5 degrees). It is possible to use all available balance holes. However, there is one extra constraint: at most one weight can be put in each balance hole. 

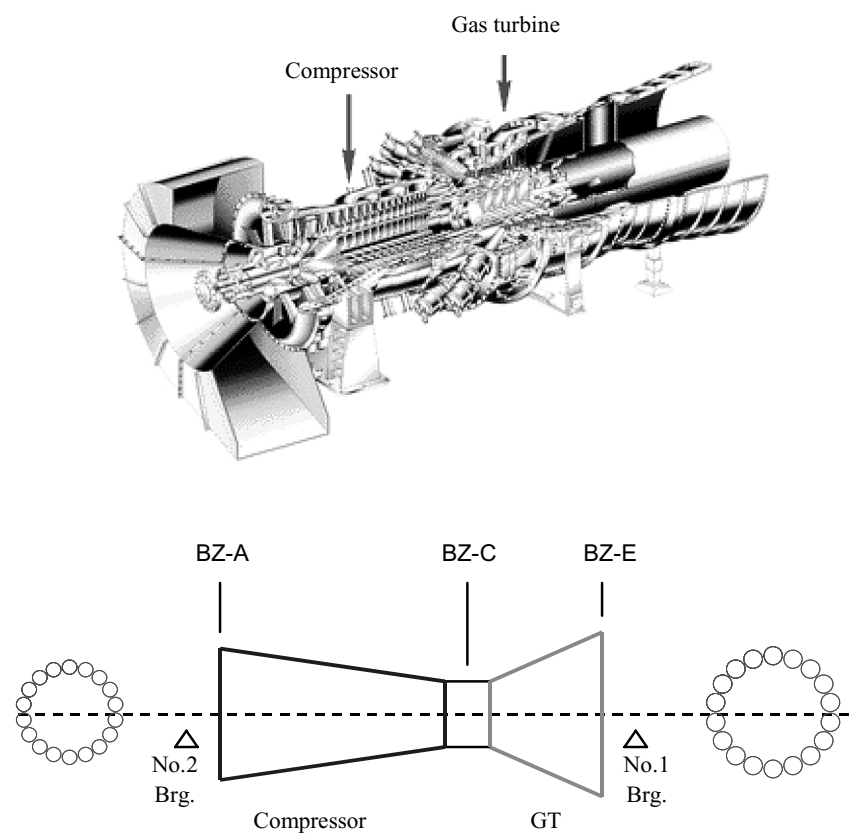

Fig. 1. Gas turbine configuration.

\subsection{Nuclear turbine}

As a more complex case, we consider the balancing of a four-rotor nuclear turbine train. It has one high pressure (HP) turbine, with a critical speed at 1,240 rpm, and three low pressure (LP) turbines, with a critical speed at $1,340 \mathrm{rpm}$. Balancing is evaluated at two different speeds, the critical speed $(1,340 \mathrm{rpm})$ and the rated speed $(1,800 \mathrm{rpm})$. The turbine configuration and vibration measuring positions are shown in Fig. 2 . We only use probes No. 3 to No. 8 because the balance weights in the LP turbine side have little effect on the vibration on the HP turbine side.

In this case, only three types of weights with weight amounts $350 \mathrm{gr}, 450 \mathrm{gr}$ and $580 \mathrm{gr}$ can be applied and each available hole can hold at most one weight. The available holes of all planes are separated by 9 degrees.

\section{Balancing methods}

Least squares and min-max balancing methods, as recalled in the appendix, are the two most commonly used balancing methods in industry under the influence coefficient framework. Their formulations can be easily converted into SOCP problems $[8,10]$ and conveniently solved by existing SOCP solvers.

\subsection{Least squares balancing}

The general least squares problem in balancing can be formulated as an SOCP problem, as shown in the appendix, as

$$
\begin{aligned}
& \operatorname{\operatorname {minimize}} \lambda, \\
& \operatorname{Re} u, \operatorname{Im} u \\
& \text { s.t. }\left\|\left[\begin{array}{l}
\operatorname{Re} A-\operatorname{Im} A \\
\operatorname{Im} A \operatorname{Re} A
\end{array}\right]\left[\begin{array}{l}
\operatorname{Re} u \\
\operatorname{Im} u
\end{array}\right]+\left[\begin{array}{l}
\operatorname{Re} v \\
\operatorname{Im} v
\end{array}\right]\right\|_{2} \leqslant \lambda,
\end{aligned}
$$

where $A$ is the influence coefficient matrix with the size $m \times n, u$ is the complex weight solution vector with the size $n \times 1, v$ is the initial vibration vector with the size $m \times 1, \lambda$ is the objective to be minimized and the optimum value of $\lambda$ represents the minimum square root of the sum of the errors. 


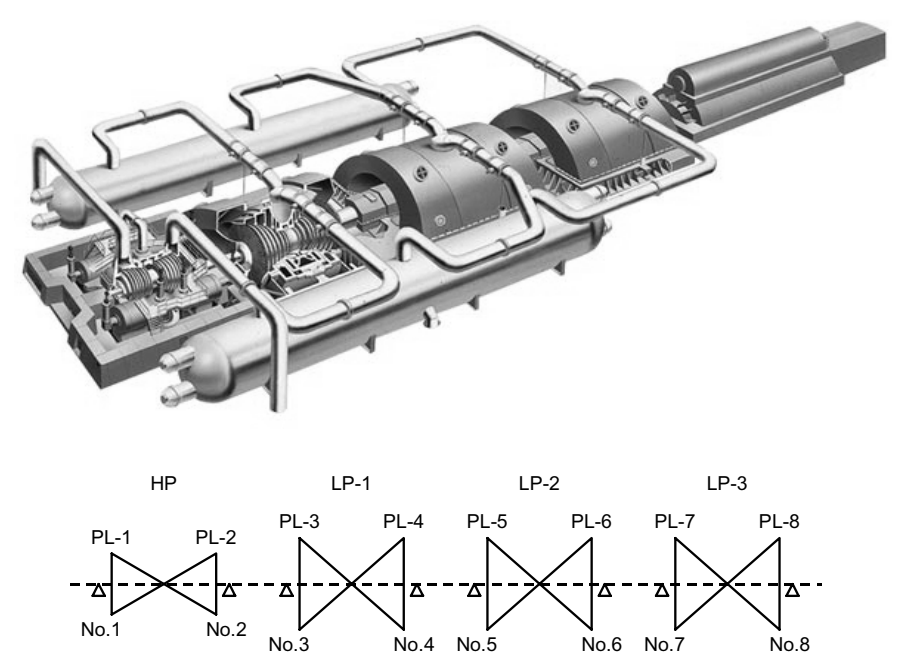

Fig. 2. Nuclear turbine configuration.

Often the balancer wishes to emphasize one or more balancing planes over others using a weighting method. The weighted least squares problem in balancing can then be formulated as an SOCP problem,

$$
\begin{aligned}
& \underset{\operatorname{Re} u, \operatorname{Im} u}{\operatorname{minime}} \lambda \\
& \text { s.t. }\left\|W\left[\begin{array}{l}
\operatorname{Re} A-\operatorname{Im} A \\
\operatorname{Im} A \operatorname{Re} A
\end{array}\right]\left[\begin{array}{l}
\operatorname{Re} u \\
\operatorname{Im} u
\end{array}\right]+W\left[\begin{array}{l}
\operatorname{Re} v \\
\operatorname{Im} v
\end{array}\right]\right\|_{2} \leqslant \lambda,
\end{aligned}
$$

where $\lambda$ is the objective to be minimized and the optimum value of $\lambda$ represents the minimum square root of the sum of the weighted errors and $W$ is the weighting matrix.

\subsection{Min-max balancing problem}

The general min-max balancing problem can be similarly formulated as

$$
\begin{aligned}
& \operatorname{minimize} \lambda, \\
& \operatorname{Re} u, \operatorname{Im} u \\
& \text { s.t. }\left\|\left[\begin{array}{l}
\operatorname{Re} a_{i}-\operatorname{Im} a_{i} \\
\operatorname{Im} a_{i} \operatorname{Re} a_{i}
\end{array}\right]\left[\begin{array}{l}
\operatorname{Re} u \\
\operatorname{Im} u
\end{array}\right]+\left[\begin{array}{l}
\operatorname{Re} v_{i} \\
\operatorname{Im} v_{i}
\end{array}\right]\right\|_{2} \leqslant \lambda, \\
& i=1,2, \cdots, m,
\end{aligned}
$$

where $\lambda$ is the objective to be minimized with the optimum value of $\lambda$ representing the minimum of the maximum value of the errors, and $a_{i}$ is the $i$-th row of the influence coefficient matrix $A$.

The general weighted min-max balancing problem can be formulated as

$$
\begin{aligned}
& \underset{\operatorname{Re} u, \operatorname{Im} u}{\min u} \lambda \\
& \text { s.t. }\left\|w_{i}\left[\begin{array}{l}
\operatorname{Re} a_{i}-\operatorname{Im} a_{i} \\
\operatorname{Im} a_{i} \operatorname{Re} a_{i}
\end{array}\right]\left[\begin{array}{l}
\operatorname{Re} u \\
\operatorname{Im} u
\end{array}\right]+w_{i}\left[\begin{array}{c}
\operatorname{Re} v_{i} \\
\operatorname{Im} v_{i}
\end{array}\right]\right\|_{2} \leqslant \lambda, \\
& i=1,2, \cdots, m,
\end{aligned}
$$

where $\lambda$ is the objective to be minimized with the optimum value of $\lambda$ representing the minimum of the maximum value of the errors and $w_{i}$ is the $i$-th diagonal element of the diagonal weighting matrix $W$. 


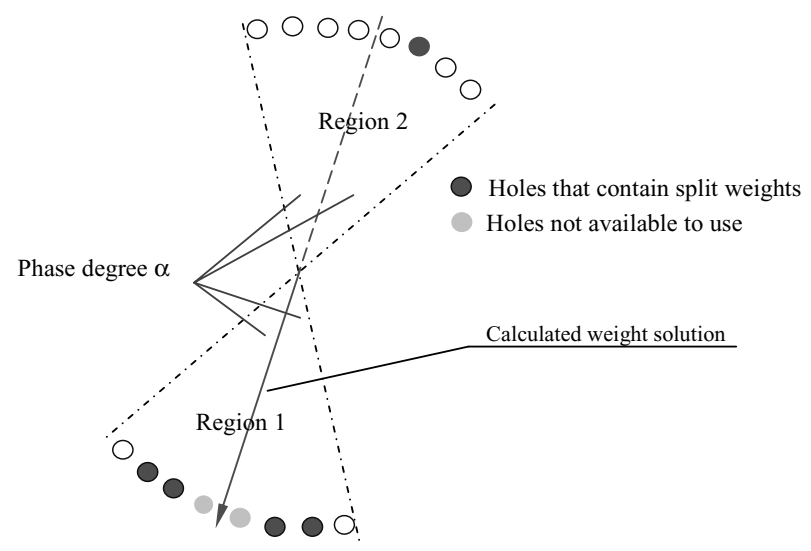

Fig. 3. Weight splitting.

\section{Practical balancing constraints}

In practice, balancers often need to consider hard practical constraints, such as maximum correction weight constraints and maximum residual response constraints. These constraints can both be cast into an SOCP formulation [8]. For additional practical balancing consideration, this paper also discusses a weight splitting constraint due to the location of existing balance weight holes and proposes an integer optimization formulation for finding an optimal weight solution that satisfies the weight splitting constraint.

\subsection{Maximum correction weight and residual response constraints}

The maximum correction weight constraints at different balance planes can be represented as

$$
\left\|\begin{array}{l}
\operatorname{Re} u_{i} \\
\operatorname{Im} u_{i}
\end{array}\right\| \leqslant w_{c_{i}}, \quad i=1,2, \cdots, n,
$$

where $w_{c_{i}}$ is the maximum correction weight that can be applied at balance plane $i$.

The maximum residual response constraints at measurement planes can be represented as

$$
\left\|\left[\begin{array}{l}
\operatorname{Re} a_{i}-\operatorname{Im} a_{i} \\
\operatorname{Im} a_{i} \operatorname{Re} a_{i}
\end{array}\right]\left[\begin{array}{l}
\operatorname{Re} u \\
\operatorname{Im} u
\end{array}\right]+\left[\begin{array}{l}
\operatorname{Re} v_{i} \\
\operatorname{Im} v_{i}
\end{array}\right]\right\|_{2} \leqslant z_{c_{i}}, i=1,2, \cdots, m,
$$

where $z_{c_{i}}$ is the desired maximum value of the vibration amplitude of the residual response entry $i$.

\subsection{Weight splitting constraint formulation}

For many practical balancing cases, there are only a limited number balancing holes and a limited number of weight sizes or types. For implementation, the calculated weight solution needs to be approximated by doing some form of weight splitting at the available holes. For each balance plane, balancers can specify a phase angle $\alpha$, which is then used to obtain the balance hole sector, such as region 1 as shown in Fig. 3. All available holes in region 1 are candidate holes to do weight splitting.

Without loss of generality, the weight splitting is assumed to be carried out at plane $i$. We suppose that there are $n_{h}$ candidate holes and $n_{w}$ types of weights available for weight splitting. For each candidate hole, there are $n_{w}$ integers related to it, representing the numbers of weights that can be placed for each weight type. It is not difficult to determine that there are $n_{h} \times n_{w}$ integers in total. We denote the integers as $x_{j k}, j=1,2, \cdots, n_{h} ; k=1,2, \cdots, n_{w}$, corresponding to different candidate holes and weight types. For flexibility, users may choose both region 1 and region 2 instead of just region 1 only, to locate candidate holes.

The mathematical formulation of weight splitting can be obtained as 


$$
\begin{array}{l|l}
\operatorname{minimize}_{\substack{x_{k}, j=1,2, \cdots, n_{h} \\
k=1,2, \cdots, n_{w}}} \| \sum_{j=1}^{n_{h}} \sum_{k=1}^{n_{w}} x_{j k} w_{j} \cos \left(\beta_{i}\right)-\operatorname{Re} u_{i} \\
n_{h=1}^{n_{h}} \sum_{k=1}^{n_{w}} x_{j k} w_{j} \sin \left(\beta_{i}\right)-\operatorname{Im} u_{i}
\end{array} \|_{2}
$$

where $w_{j}$ is the amount of weight for weight type $j$ and $\beta_{i}$ is the phase of the $i$-th candidate hole related to the calculated weight solution line.

Other weight splitting constraints can also be imposed by the user.

\subsection{Maximum weights per hole and maximum number of hole constraints}

The constraint of the maximum number of weights per hole is expressed as

$$
\sum_{k=1}^{n_{w}} x_{j k} \leqslant \text { max. number of weights per hole, } \quad j=1,2, \cdots, n_{h} .
$$

The constraint on the maximum number of holes that contain the split weights is formulated as

$$
\sum_{j=1}^{n_{w}} 1\left(\sum_{k=1}^{n_{w}} x_{j k}\right) \leqslant \text { max. number of holes that contain split weights, }
$$

where $1: R \rightarrow R$ is the unit step function. It generates 1 when the input is positive and 0 otherwise.

The weight splitting optimization problem Eq. (7) with practical weight splitting constraints can be solved more efficiently by using existing Integer Programming (IP) solvers. However, if the problem size is tolerable and the computation time is not an issue, we can also obtain the optimal weight splitting solution by searching every possible combination of those integer variables. This works for most industrial balancing applications and no IP optimization software is needed.

\section{Balancing results}

In each balancing case, original vibrations were measured and then trial weights were used to evaluate the influence coefficients. The strict plane constraints need to be satisfied as well. In actual field balancing, the weighted least squares method is usually employed to balance the rotor. This section presents a balancing simulation using the min-max balancing method as described in Sections 3 and 4 to calculate new balance weights and the resulting vibration using the same influence coefficients.

\subsection{Case 1: Gas turbine}

For this specific application, without weight splitting, the min-max method and the least squares method both generate the same balance weight solution. However, when applying the weight splitting as shown in Eq. (7), the min-max balance method generates better results than the actual balancing results.

The measured baseline vibration is

$$
v=\left[\begin{array}{c}
32 \mu \mathrm{m} \angle 357^{\circ} \\
105 \mu \mathrm{m} \angle 346^{\circ}
\end{array}\right]
$$

In planes BZ-A and BZ-E, the influence coefficient matrix was obtained from field trial testing as

$$
A=\left[\begin{array}{ll}
0.085 \mu \mathrm{m} / \mathrm{g} \angle 27^{\circ} 0.05 \mu \mathrm{m} / \mathrm{g} \angle 82^{\circ} \\
0.053 \mu \mathrm{m} / \mathrm{g} \angle 57^{\circ} 0.071 \mu \mathrm{m} / \mathrm{g} \angle 15^{\circ}
\end{array}\right] .
$$

The equivalent weight solution after considering the weight splitting constraint for the min-max method is calculated as 


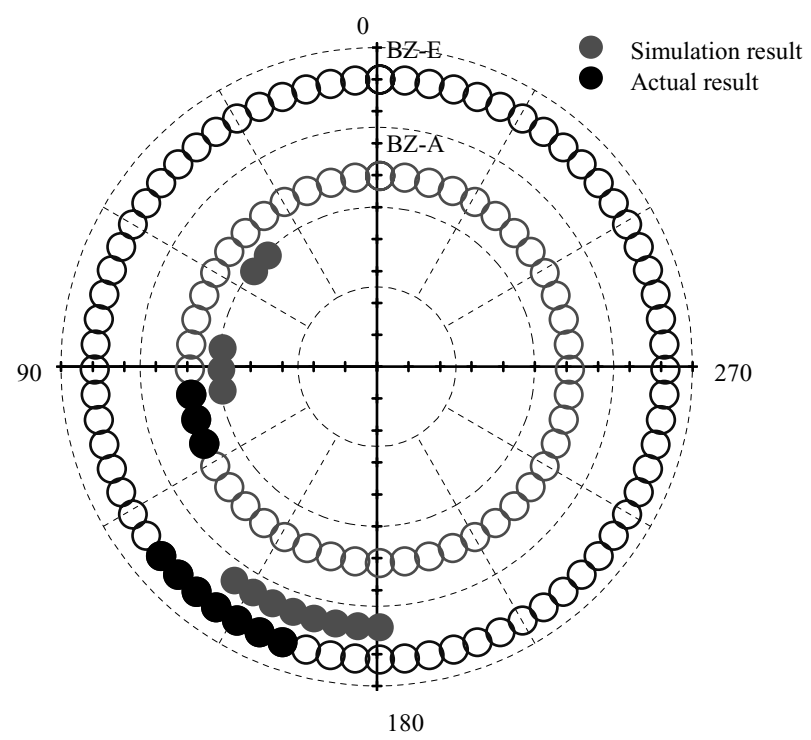

Fig. 4. Comparison of balance weight distribution.

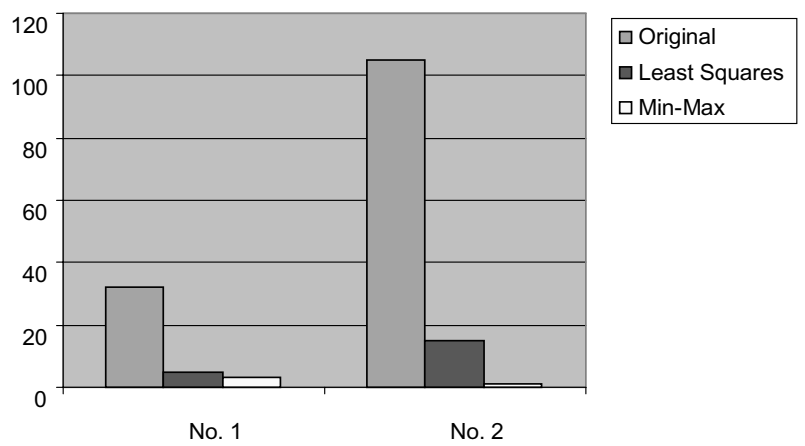

Fig. 5. Amplitude comparision for the gas turbine: least squares vs min-max.

$$
n=\left[\begin{array}{c}
663 \mathrm{~g} \angle 74^{\circ} \\
1104 \mathrm{~g} \angle 166^{\circ}
\end{array}\right]
$$

This equivalent weight solution predicts a resulting vibration at the level of

$$
v_{u}=A u=\left[\begin{array}{c}
32 \mu \mathrm{m} \angle 172^{\circ} \\
104 \mu \mathrm{m} \angle 166^{\circ}
\end{array}\right] \text {. }
$$

The final vibration will be

$$
z=v+v_{u}=\left[\begin{array}{l}
3 \mu \mathrm{m} \angle 85^{\circ} \\
1 \mu \mathrm{m} \angle 346^{\circ}
\end{array}\right] .
$$

In applying the calculated balance weight solution in the gas turbine, a weight splitting procedure is followed after obtaining a calculated solution. For many industrial high-speed rotating machines, there are only a limited number of balance holes and usually only a few weight types are available to use. The weight solution for the min-max simulation using Eqs (3) (7), (8) and (9) is shown together with the actual least squares weight solution in Table 1. The weight distributions for the two balancing methods are shown in Fig. 4.

The original vibration, weighted least squares balancing with weight splitting (without optimization) and min-max simulation results with weight splitting are given in Table 2. The amplitude comparison of these balancing results is 
Table 1

Comparison of weight distribution for the gas turbine

\begin{tabular}{lccccc}
\hline Plane no. & \multicolumn{2}{c}{ BZ-A } & & \multicolumn{2}{c}{ BZ-E } \\
\cline { 2 - 3 } \cline { 5 - 6 } Actual (least squares) & gr & deg & & gr & deg \\
\hline & 142 & 97.5 & & 142 & 130 \\
& 142 & 105 & & 142 & 135 \\
& & & & 142 & 145 \\
& & & & 142 & 150 \\
Simulation (min-max) & 142 & 45 & & 142 & 145 \\
& 142 & 52.5 & & 142 & 150 \\
& 142 & 82.5 & & 142 & 155 \\
& 142 & 90 & & 142 & 165 \\
& 142 & 97.5 & & 142 & 170 \\
& & & & 142 & 175 \\
& & & & 142 & 180 \\
& & & & 142 & 185 \\
\hline
\end{tabular}

Table 2

Vibration conditions for the gas turbine

\begin{tabular}{llcccccc}
\hline & Probe no. & & \multicolumn{2}{c}{ No.1(BZ-A) } & & \multicolumn{2}{c}{ No.2(BZ-E) } \\
\cline { 1 - 2 } \cline { 7 - 8 } Speed & Condition & & Amp & deg & & Amp & deg \\
\hline \multirow{3}{*}{$3000 \mathrm{rpm}$} & Original & & 32 & 357 & & 105 & 346 \\
& Least squares & & 5 & 150 & & 15 & 210 \\
& Min-max & & 3 & 85 & & 1 & 346 \\
\hline
\end{tabular}

Unit: $\mu \mathrm{mP}-\mathrm{P}$.

Table 3

Original vibration conditions

\begin{tabular}{clcccccc}
\hline Probe no. & & No.3 & No.4 & No.5 & No.6 & No.7 & No.8 \\
\hline Critical speed & Amp & 30 & 15 & 78 & 85 & 74 & 51 \\
& deg & 210 & 160 & 9 & 4 & 177 & 175 \\
Rated speed & Amp & 25 & 16 & 15 & 31 & 58 & 23 \\
& deg & 260 & 105 & 145 & 26 & 235 & 52 \\
\hline
\end{tabular}

Unit: $\mu \mathrm{mP}-\mathrm{P}$.

shown in Fig. 5. It can be seen that the min-max balancing method with the proposed weight splitting leads to much better balancing results. In particular, the final min-max result shows a vibration reduction from 5 to $3 \mu \mathrm{mP}-\mathrm{P}$ in sensor plane 1 and from 15 to $1 \mu \mathrm{mP}-\mathrm{P}$ in plane 2 . Thus the maximum vibration level was reduced from $15 \mu \mathrm{mP}-\mathrm{P}$ to $3 \mu \mathrm{mP}-\mathrm{P}$ for the gas turbine case or an $80 \%$ reduction in the maximum vibration level.

\subsection{Case 2: Nuclear turbine}

As described in Section 2, the balancing of the four-rotor nuclear turbine involves two different speeds, the critical speed and the rated speed, five sensors, and probes No. 3 to No. 8. The original vibration condition is shown in Table 3.

The field balancing utilized the weighted least squares method with special field balancing constraints considered. To illustrate how the min-max balancing can help to improve the balancing performance, three different types of simulation using the min-max method were applied sequentially. First the min-max balancing without considering any practical weight constraints was tried. The min-max method without constraints can achieve the minimum of the largest residual vibration at all speeds and all sensor locations. However, at the rated speed, the resulting vibration level turned out to be worse than the original least squares results. To guarantee that the vibration level is lower than the required value at the rated speed, in the second simulation, the residual response constraint at the rated speed 
Table 4

Vibration with least squares balancing and min-max 1 balancing for the nuclear turbine

\begin{tabular}{|c|c|c|c|c|c|c|c|c|}
\hline & \multicolumn{2}{|c|}{ Probe no. } & No.3 & No.4 & No.5 & No.6 & No.7 & No.8 \\
\hline \multirow{4}{*}{ Least squares } & Critical speed & Amp & 19 & 7 & 63 & 65 & 35 & 32 \\
\hline & & deg & 239 & 187 & 23 & 13 & 210 & 226 \\
\hline & Rated speed & Amp & 14 & 13 & 8 & 14 & 8 & 7 \\
\hline & & deg & 274 & 93 & 100 & 70 & 270 & 323 \\
\hline \multirow[t]{4}{*}{ Min-max_1 } & Critical speed & Amp & 28 & 19 & 29 & 29 & 7 & 26 \\
\hline & & deg & 328 & 322 & 40 & 343 & 272 & 243 \\
\hline & Rated speed & Amp & 29 & 11 & 13 & 29 & 29 & 22 \\
\hline & & deg & 54 & 218 & 136 & 307 & 83 & 288 \\
\hline
\end{tabular}

Unit: $\mu \mathrm{mP}-\mathrm{P}$.

Table 5

Comparison of weight distributions for the nuclear turbine

\begin{tabular}{lccccccccc}
\hline Plane no. & \multicolumn{2}{c}{ PL-4 } & & \multicolumn{2}{c}{ PL-5 } & & \multicolumn{2}{c}{ PL-8 } \\
\cline { 2 - 3 } & gr & deg & & gr & deg & & gr & deg \\
\hline Least squares & 350 & 270 & & 580 & 81 & & 580 & 252 \\
& & & & & & & 450 & 261 \\
Min-max_1 & 1178 & 280 & & 1441 & 117 & & 1672 & 277 \\
\hline
\end{tabular}

Unit: $\mu \mathrm{mP}-\mathrm{P}$

Table 6

Rotor vibration from min-max_2 with the response constraint for the nuclear turbine

\begin{tabular}{lllccccccc}
\hline & \multicolumn{2}{c}{ Probe no. } & & No.3 & No.4 & No.5 & No.6 & No.7 & No.8 \\
\hline Min-max_2 & Critical speed & Amp & 15 & 10 & 51 & 49 & 26 & 36 \\
& & deg & 311 & 331 & 30 & 10 & 221 & 222 \\
& \multirow{2}{*}{ Rated speed } & Amp & 10 & 1.3 & 8.2 & 10 & 6.9 & 10 \\
& & deg & 69 & 42 & 97 & 321 & 31 & 288 \\
\hline
\end{tabular}

Unit: $\mu \mathrm{mP}-\mathrm{P}$.

was employed in the min-max balancing formulation. Finally, to apply the calculated balance weight in this field balancing, we still need to do weight splitting on the basis of the second simulation. That is the third simulation we have conducted.

In summary, three different types of min-max balancing simulation were carried out.

- Simulation 1 (min-max_1): without any constraints;

- Simulation 2 (min-max 2): $10 \mu \mathrm{mP}-\mathrm{P}$ as the maximum vibration at the rated speed;

- Simulation 3 (min-max 3): $10 \mu \mathrm{mP}-\mathrm{P}$ as the maximum vibration at the rated speed with weight splitting.

Table 4 gives the final vibration results and Table 5 shows the weight distributions. These results show that the min-max method requires much more weight to reduce the vibration at the critical speed. It is possible to add a maximum balancing weight constraint to the min-max balancing. However, in industry, much more attention is paid to the vibration at the rated speed. We assigned the maximum vibration $(10 \mu \mathrm{mP}-\mathrm{P})$ constraint at the rated speed to obtain a better solution. With the new constraint being considered, the residual vibration for the least squares balancing and the prediction from the min-max method are summarized in Table 6.

To obtain the balance solution that can be used in the final min-max balancing, the weight splitting function is applied as well. Table 7 shows the two different weight distributions and Fig. 6 gives a graphical comparison.

Table 8 gives the final amplitude and phase results for the final min-max 3 balancing results at the critical speed and at the rated speed. Figure 7 shows the comparison between the original vibration, least squares and min-max 3 with constraints. The peak response at sensor location 6 is reduced from $63 \mu \mathrm{mP}-\mathrm{P}$ with the least squares method to $51 \mu \mathrm{mP}-\mathrm{P}$ with min-max 3 at the critical speed. At the rated speed, the maximum least squares vibration was $15 \mu \mathrm{mP}-\mathrm{P}$ at sensors 3 and 6 while the maximum value from min-max 3 was $10 \mu \mathrm{mP}-\mathrm{P}$ at sensors 3 and 8 . This is a $33 \%$ reduction in vibration at the rated speed.

From this result, it is apparent that the min-max method reduced the maximum vibration level very significantly. 
Table 7

Comparison of weights distribution for the nuclear turbine

\begin{tabular}{ccccccccc}
\hline Plane no. & \multicolumn{2}{c}{ PL-4 } & & \multicolumn{2}{c}{ PL-5 } & & \multicolumn{2}{c}{ PL-8 } \\
\cline { 2 - 3 } & gr & deg & & gr & deg & & gr & deg \\
\hline Least squares & 350 & 270 & & 580 & 81 & & 580 & 252 \\
& & & & & & 450 & 261 \\
Min-max_3 & 580 & 270 & & 580 & 90 & & 350 & 234 \\
& 350 & 315 & & 350 & 117 & & 580 & 261 \\
& & & & & & 450 & 288 \\
\hline
\end{tabular}

Unit: $\mu \mathrm{mP}-\mathrm{P}$

Table 8

Min-max_3 final vibration with the response constraint and weight splitting

\begin{tabular}{lllccccccc}
\hline & \multicolumn{2}{c}{ Probe no. } & & No.3 & No.4 & No.5 & No.6 & No.7 & No.8 \\
\hline Min-max_3 & Critical speed & Amp & 15 & 10 & 51 & 50 & 6 & 36 \\
& & $\operatorname{deg}$ & 313 & 331 & 29 & 10 & 221 & 222 \\
& \multirow{2}{*}{ Rated speed } & Amp & 10 & 0.4 & 8 & 9 & 7 & 10 \\
& & deg & 71 & 15 & 94 & 326 & 36 & 289 \\
\hline
\end{tabular}

Unit: $\mu \mathrm{mP}-\mathrm{P}$

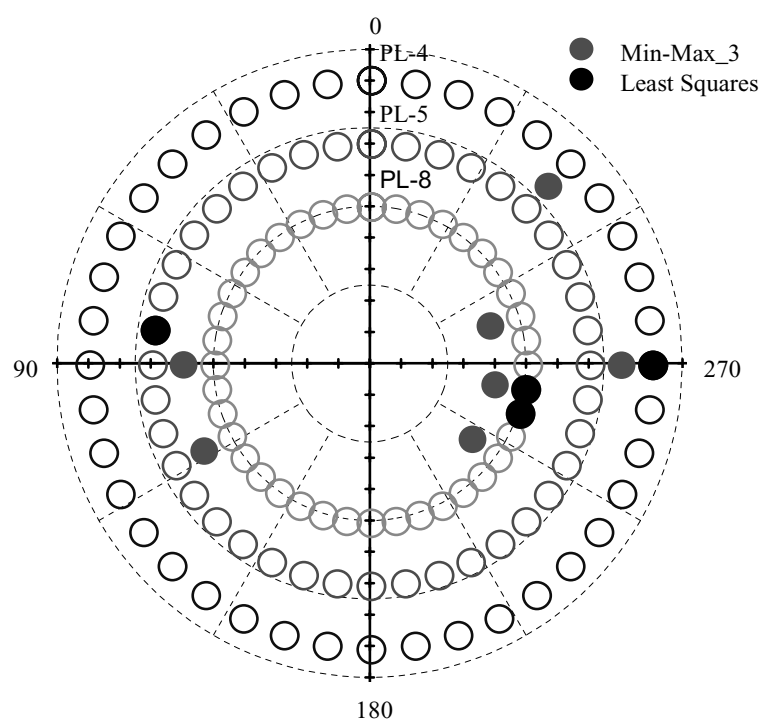

Fig. 6. Comparison of balance weight distribution.

\section{Conclusions}

This paper reported on the balancing of two industrial rotors from Mitsubishi Heavy Industrials Litd. Both the least squares formulation and the min-max formulation utilizing the second-order cone programming (SOCP) were adopted. The reported balancing approach is expected to have significant impact practical applications as it allows the consideration of various practical constraints such as the maximum weight constraint, the maximum residual response constraint, and the weight splitting constraint. Our immediate effort is focused on the field trial testing of the proposed balancing approach.

\section{Acknowledgment}

The authors acknowledge the support of the Rotating Machinery and Controls (ROMAC) Laboratory at the University of Virginia, and its member companies for their support of this project. 


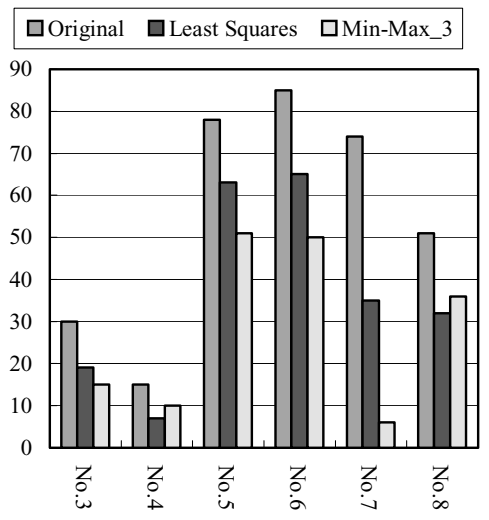

At critical speed

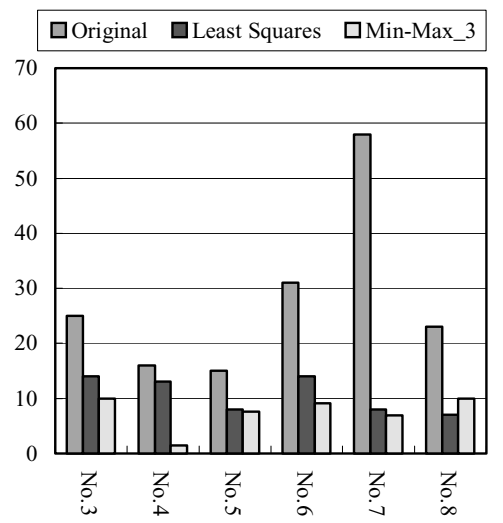

At rated speed

Fig. 7. Amplitude comparison: Original, least squares and min-max 3 balancing results.

\section{Appendix}

\section{A-1. Influence coefficient framework}

If the rotor response to the unbalance force is linear, and the rotor run out as well as the shaft bow effects are compensated, the balancing problem can be represented in the short hand complex notation form as follows,

$$
z=A u+v \text {. }
$$

Here $u \in \mathrm{C}^{n}$ is the correction weight vector, $v \in \mathrm{C}^{m}$ and $z \in \mathrm{C}^{m}$ are the vibration measurement vectors representing the initial vibration and residual response at particular sensor locations and rotation speeds.

The influence coefficient matrix $A \in \mathrm{C}^{m \times n}$ represents the vibration measured at the selected speeds. It is usually obtained by experimental estimation using trial weights. The objective of balancing is to minimize $z$ according to certain criteria under certain additional balancing constraints.

We consider methods of evaluating the final response $z$. The $l_{p}$ vector norm can be selected as the objective function. The fundamental balancing principle is

$$
\text { Minimize }\|W z\|_{p},
$$

where $W$ represents the weighting matrix. The $l_{p}$ norm is defined as

$$
\|x\|_{p}:=\left\{\begin{array}{c}
\left(\sum_{i=1}^{n}\left|x_{i}\right|^{p}\right)^{\frac{1}{p}}, p \in[1, \infty) ; \\
\max _{i}\left|x_{i}\right|, p=\infty .
\end{array}\right.
$$

Important cases for balancing include: 1) $l_{1}$ minimization or Least Absolute Deviation (LAD), 2) $l_{2}$ minimization or least-squares (LS), and 3) $l_{\infty}$ or Min-Max (Chebyshev), corresponding to $p=1,2$ and $\infty$, respectively. The least squares method has some nice features, such as its simple form and existing analytical solution. However, the min-max method is better if the objective is to minimize the maximum vibration amplitude in the rotor.

Second order cone programming is one important category of convex optimization. It contains Linear Programming (LP) and Quadratic Programming (QP) as special cases. One advantage of using SOCP is that it enables users to cast balancing formulation as well as practical constraints into second order cones without having to use any approximation. The converted SOCP problem can then be solved by efficient polynomial algorithms.

\section{A-2. Second order cone programming}

The general formulation for a second order cone programming problem is

minimize $f^{\mathrm{T}} x$

subject to $\left\|A_{i} x+b_{i}\right\|_{2} \leqslant c_{i}^{\mathrm{T}} x+d_{i}, i=1,2, \cdots, N$.

This formulation is employed in this paper for the solution of the industrial balancing problem. 


\section{References}

[1] M.S. Darlow, Balancing of High Speed Machinery, Springer, New York, 1989.

[2] W.C. Foiles, P.E. Allaire and E.J. Gunter, Review: rotor balancing, Shock and Vibration 5 (1998), 325-336.

[3] T.P. Goodman, A least-squares method for computing balance corrections, Journal of Engineering for Industry 86 (1964), $273-279$.

[4] R.M. Little and W.D. Pilkey, A linear programming approach for balancing flexible rotors, Journal of Engineering for Industry 98(3) (1976), 1030-1035.

[5] E. Woomer and W.D. Pilkey, The balancing of rotating shafts by quadratic programming, Journal of Mechanical Design 103 (1981), 831-834.

[6] H. Kanki, M. Kawanishi and K. Ono, Anew balancing method applying LMI optimization method, Proceddings of $5^{\natural}$ International Conference on Rotor Dynamics, Darmstadt, Germany, 1998, 667-678.

[7] S. Boyld and L. Vandenberghe, Convex Optimization, Cambridge University Press, 2004.

[8] G. Li, Z. Lin and P.E. Allaire, Robust optimal balancing of high-speed machinery using convex optimization, ASME Journal of Vibration and Acoustics (2007), to appear.

[9] A. El-Shafei, A.S. El-Kabbany and A.A. Younan, Rotor Balancing without Trial Weights, Journal of Engineering for Gas Turbines and Power 126(3) (2004), 604-609.

[10] M.S. Lobo, L. Vandenberghe, S. Boyd and H. Lebret, Applications of second-order cone programming, Linear Algebra And Its Applications 284 (1998), 193-228. 

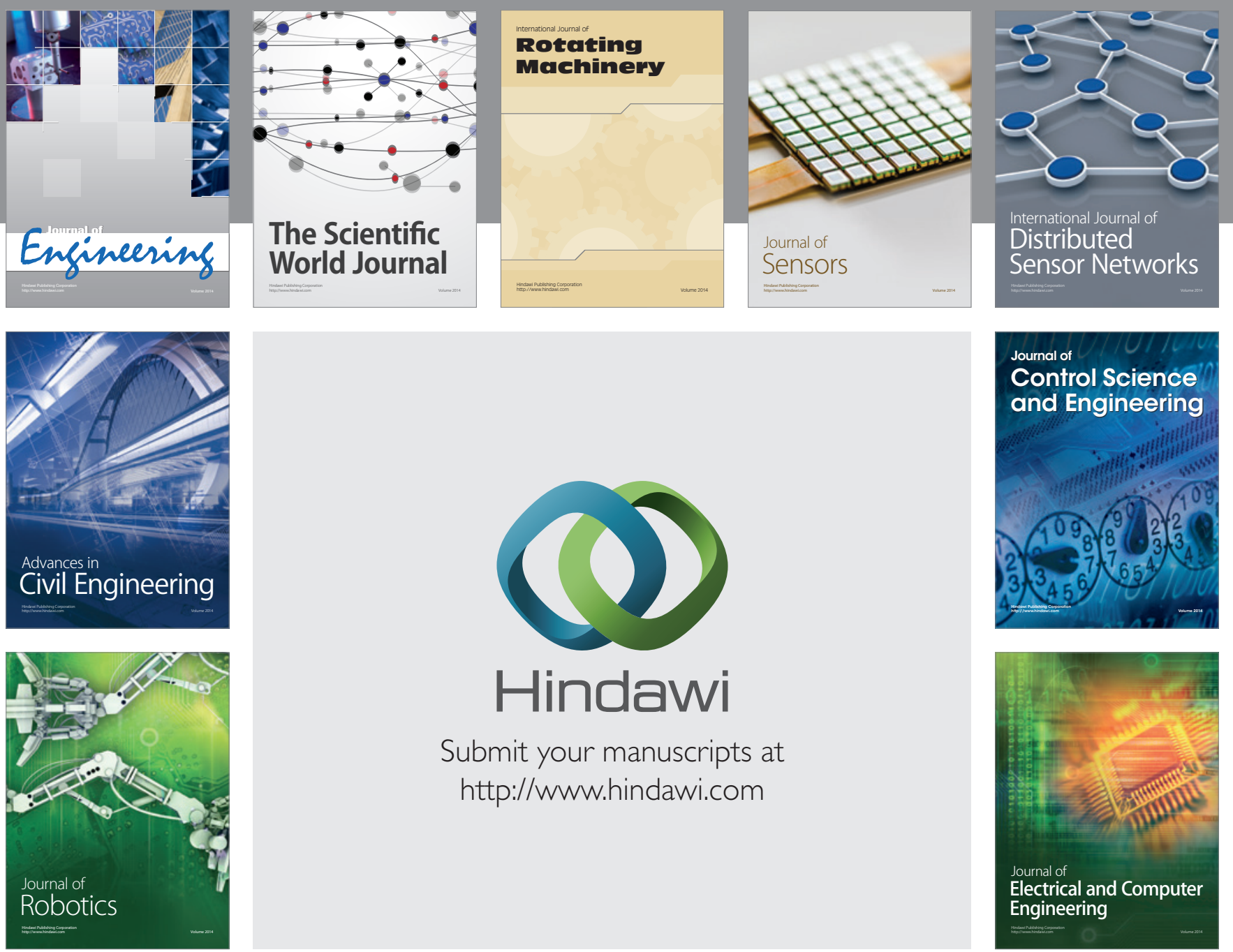

Submit your manuscripts at

http://www.hindawi.com
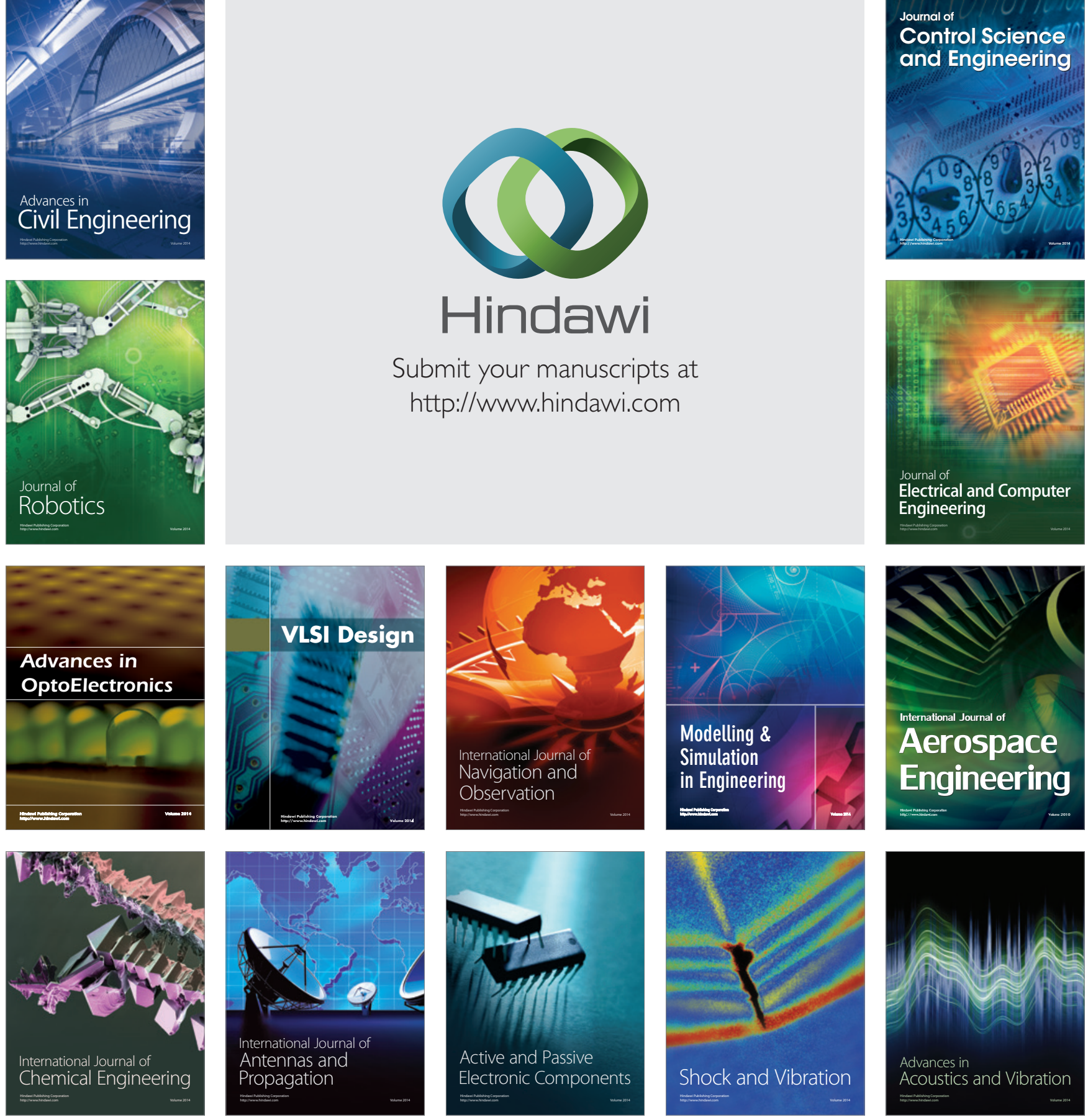